\title{
Applications of Voltammetry in Lithium Ion Battery Research
}

\author{
Taewhan Kim ${ }^{1 \dagger}$, Woosung Choi $^{1 \dagger}$, Heon-Cheol Shin², Jae-Young Choi ${ }^{3,4}$, Ji Man Kim, Min-Sik Park ${ }^{6}$, \\ and Won-Sub Yoon ${ }^{1 *}$ \\ ${ }^{1}$ Department of Energy Science, Sungkyunkwan University, Suwon 16419, South Korea \\ ${ }^{2}$ School of Materials Science and Engineering, Pusan National University, Busan 46241, Republic of Korea \\ ${ }^{3}$ School of Advanced Materials Science and Engineering, Sungkyunkwan University, Suwon, 16419, Republic of Korea \\ ${ }^{4}$ SKKU Advanced Institute of Nanotechnology (SAINT), Sungkyunkwan University, Suwon 16419, Republic of Korea \\ ${ }^{5}$ Department of Chemistry, Sungkyunkwan University, Suwon, 16419, South Korea \\ ${ }^{6}$ Department of Advanced Materials Engineering for Information and Electronics, Kyung Hee University, Yongin, 17104, \\ Republic of Korea
}

\begin{abstract}
$\mathrm{Li}$ ion battery (LIB) is one of the most remarkable energy storage devices currently available in various applications. With a growing demand for high-performance batteries, the role of electrochemical analysis for batteries, especially, electrode reactions are becoming very important and crucial. Among various analytical methods, cyclic voltammetry $(\mathrm{CV})$ is very versatile and widely used in many fields of electrochemistry. Through CV, it is possible to know electrochemical factors affecting the reaction voltage and reversibility, and furthermore, quantitative analysis on $\mathrm{Li}^{+}$diffusivity as well as intercalation and capacitive reactions, and also anionic redox reaction. However, the explanation or interpretation of the results of $\mathrm{CV}$ is often deficient or controversial. In this mini-review, we briefly introduce the principle of cyclic voltammetry and its applications in LIB to bring a better understanding of the electrochemical reaction mechanisms involved in LIB.
\end{abstract}

Keywords : Li Ion Battery, Cyclic Voltammetry, Electrochemistry

Received : 4 November 2019, Accepted : 12 November 2019

\section{Introduction}

Li ion battery has the best performance among rechargeable batteries that are currently in commercial use and is widely used in portable products such as mobile phones, laptops, power tools, and electric vehicles. In addition, due to technological advances, the performances required for $\mathrm{Li}$ ion batteries such as high capacity long cycle life have been gradually enhanced [1-6]. Looking at the Li ion batteries, electrochemical reactions take place in the electrodes, and even in the electrolyte. To analyze these electrochemical processes, various electrochemical analysis are being studied. Commonly used electrochemical methods include cyclic voltammetry (CV), chrono-

$\dagger$ These author contributed equally to this work

*E-mail address: wsyoon@skku.edu

DOI: https://doi.org/10.33961/jecst.2019.00619

This is an open-access article distributed under the terms of the Creative Commons Attribution Non-Commercial License (http://creativecommons.org/licenses/by-nc/4.0) which permits unrestricted non-commercial use, distribution, and reproduction in any medium, provided the original work is properly cited. amperometry (CA) [7], chronopotentiometry (CP) [8], stripping voltammetry (SV) [9], and linear sweep voltammetry (LSV) [10]. It is possible to know the voltage at which oxidation or reduction occurs, and whether impurities are present or whether the reaction has occurred properly. These electrochemical analyses are very useful in a variety of fields, especially energy storage systems like Li ion batteries. Cyclic voltammetry has long been a fundamental analytical technique among electrochemical methods. The advantages of CV compared to other measurements are: 1) It is possible to know whether the chemical reaction of the reactants is reversible or irreversible. 2) The potential at which an oxidation or reduction reaction occurs can be determined. 3) Quantitative analysis is also possible for substances whose concentration is not known. 4) It is possible to measure by changing test conditions such as scan rate, temperature and concentration of reactant. In this mini-review, the basic principles and applications of $\mathrm{CV}$ for $\mathrm{Li}$ ion batteries research are introduced. 

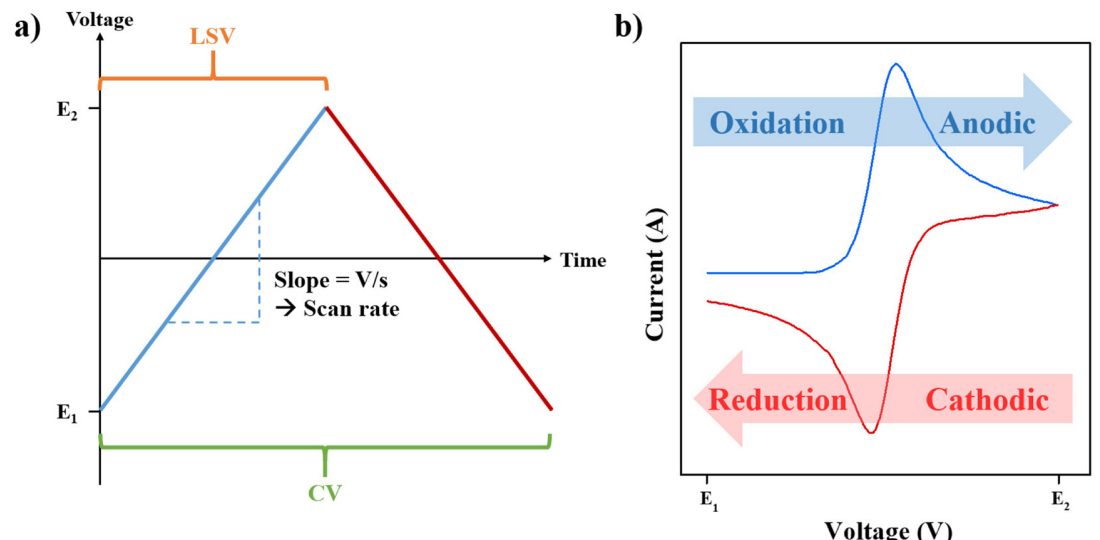

Fig. 1. a) Voltage vs. time profile for linear sweep and cyclic voltammetry. b) Typical cyclic voltammogram plotting cathodic and anodic peak.

\section{Principle of voltammetry}

\subsection{Linear sweep and cyclic voltammetry}

First, cyclic voltammetry is based on the principle of linear sweep voltammetry that is a technique to measure the current while the potential is swept linearly as a function of time. Here, the slope of the voltage change over time is defined as a scan rate $\left(\mathrm{m} \mathrm{s}^{-1}\right)$. LSV shows various results depending on the following factors: 1 ) the rate of electron transfer process, 2) the electrochemical reactivity of the materials, and 3 ) the scan rate for measurement. The results of LSV are presented as one of anodic or cathodic peak as described in Fig. 1a. Although CV is very similar to LSV, it does not finish with a single sweep within a fixed voltage range. For example, assuming the voltage range from $E_{1}$ to $E_{2}$, when $E_{2}$ is reached at the end, it reverses to $E_{1}$ again. Because of this feature, it is called 'cyclic' current versus voltage plot is termed a 'cyclic voltammogram', as shown in Fig. 1b. In the CV measurements, the most important parameter is the scan rate $(v)$. The voltage is swept from $E_{1}$ to $E_{2}$ and it represents the slope for a linear voltage change during the measurement. By repeating several cycles with the same conditions, additional information on the voltage, reversibility, and cyclability of redox reactions can be obtained. Also, it can be used to investigate the kinetics of electrochemical redox reaction by changing the scan rate.

\subsection{Comparison of cyclic voltammogram and $d Q$ $d V$ plot}

For differential capacity $(d Q / d V)$ plot, it can be recorded based on a similar principle with cyclic voltammogram. Although it provides useful information on the redox reaction of electrochemical systems the difference between cyclic voltammogram and $d Q / d V$ plot should be recognized as cyclic voltammogram contains the rate information. In general, the current (I) is plotted on the y-axis versus the voltage (V) on the $\mathrm{x}$-axis in the cyclic voltammogram and it can be transformed into to $d Q / d V$ plot. For transformation of cyclic voltammogram to $d Q / d V$, the I and $\mathrm{V}$ can be expressed as

$$
\begin{aligned}
& V=v t \\
& d t=\frac{d V}{v} \\
& I=\frac{d Q}{d t}
\end{aligned}
$$

where $v$ is scan rate $\left(\mathrm{V} \mathrm{s}^{-1}\right), V$ is the voltage (or potential, V), $I$ is the current (A), $Q$ is charge stored $(\mathrm{Ah})$, and $\mathrm{t}$ is time (s). And the formula (4) which indicate the $\mathrm{y}$-axis of $d Q / d V$ plot was obtained by combining the equation (2) and (3)

$$
\frac{I}{v}=\frac{d Q}{d V}
$$

Moreover, it is necessary to understand the different experimental conditions between galvanostatic cycling (charge-discharge) and CV in terms of a driving force for electrochemical reactions; the potential increases linearly with time in $\mathrm{CV}$, whereas the current is maintained during galvanostatic cycling. 
Regardless of state of charge (SOC) of the working electrode, these forced changes in potential increase the current beyond the operating limits of the cell. Thus, it would be difficult to make a comparison of accessible capacity [11]. In addition, the IR drop of the cell remains consistent during the constant current scan, which depends on the CV sweep, so the peak position and shape can be changed as shown in Fig. 2.

Therefore, before using $d Q / d V$ plot, it is necessary to understand the commonalities and differences between $\mathrm{CV}$ and $d Q / d V$.

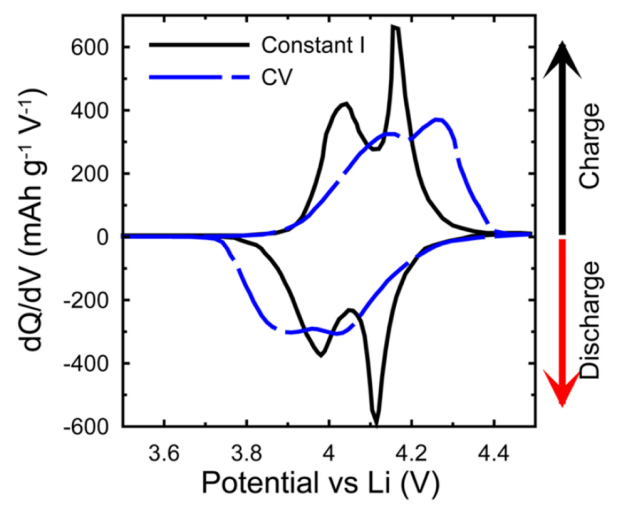

Fig. 2. $\mathrm{CV}$ and $\mathrm{dQ} / \mathrm{dV}$ curve of $\mathrm{Li}_{\mathrm{x}} \mathrm{Mn}_{2} \mathrm{O}_{4+\delta}$ vs $\mathrm{Li}$ metal with constant current experiment as $\mathrm{C} / 8=0.33 \mathrm{~mA} / \mathrm{cm}^{2}$ and $\mathrm{CV}: 0.04 \mathrm{mV} / \mathrm{S}$ (sweeps up and down in $6.9 \mathrm{~h}$ ). Reprinted with permission from Ref. [12]. Copyright (C) 2007 by Taylor \& Francis Group, LLC.

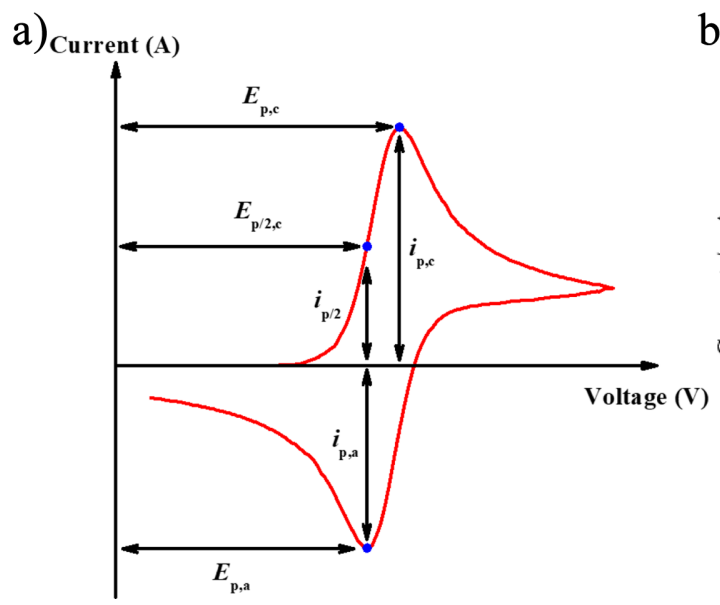

\subsection{Equations of voltammetry for intercalation system}

Fig. 3 shows a unique cyclic voltammogram that is distinguished by reversible, quasi-reversible and irreversible processes depending on the rate of charge transfer. One of the ways to distinguish each process is checking the change of cyclic voltammogram with various scan rates. Matsuda and Ayabe suggested the zone boundaries of reversible, quasi-reversible, and totally irreversible systems [14]. In a reversible processes, since the rate of electron transfer is rapid enough at the surface of electrode, the peak potential $E_{\mathrm{p}}$ is independent on the variation of scan rate $v$, and anodic $\left(E_{p, a}\right)$ and cathodic $\left(E_{p, c}\right)$ peak potential separation, is $59.0 / n \mathrm{mV}, i_{p, a} / i_{p, c}=1$, in addition, the following equation is satisfied.

$$
\left|E_{p}-E_{p / 2}\right|=2.20 \frac{R T}{n F}=\frac{56.5}{n} m V \text { at } 298 K
$$

Unlikely, in a quasi-reversible process which show limitation of electron transfer kinetics, the peak potential is a function of scan rate. $\mathrm{Li}^{+}$intercalation reactions tend to follow a quasi-reversible process since cathodic and anodic peaks are dependent on the scan rate [15]. The cyclic voltammogram of quasireversible process shows gradual peak shifts to higher potential value with an increase of scan rate than one of reversible process due to the overpotential.

The magnitude of the current $I_{p}^{R e v}$ is expressed by the Randles-Sevcik equation for a reversible electron transfer process (at $298 \mathrm{~K}$ ):

b)

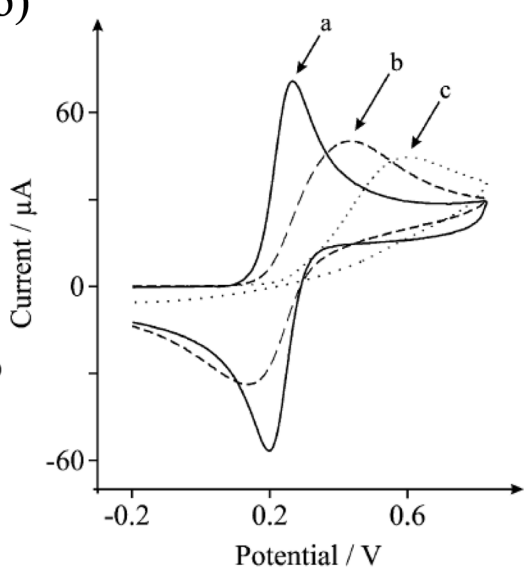

Fig. 3. a) An example of cyclic voltammogram and b) cyclic voltammogram in case of reversible, quasi-reversible and irreversible electron transfer. Reprinted with permission from Ref.[13]. Copyright (C) 2012 The Royal Society of Chemistry. 


$$
I_{p}^{R e v}= \pm\left(2.69 \times 10^{5}\right) n^{3 / 2} A C D^{1 / 2} v^{1 / 2}
$$

where $n$ is the total number of electrons reacted per mole in the electrochemical process, $\mathrm{A}$ is the electrode area $\left(\mathrm{cm}^{2}\right), \mathrm{C}$ is the concentration of the electrode material at surface, and D is the diffusion coefficient.

However, above equation (6) would be difficult to adapt in the case of intercalation compounds for LIB. To obtain exact $\mathrm{Li}^{+}$ion diffusivity, two boundary conditions should be considered according to the scan rate; i) a semi-infinite diffusion process (a fast scan rate) and ii) a finite diffusion process (a slow scan rate). In the case of the semi-infinite diffusion process, the peak current is proportional to the square root of scan rate. In contrast, the peak current is proportional to scan rate in the finite diffusion process, in which the anodic/cathodic reaction can be characterized by the following equation;

$$
I_{p}=\frac{n^{2} F^{2} v l A C}{2 R T}
$$

Considering the different diffusion behaviors induced by the scan rate, Aoki et al. generalized the equations for peak currents at all scan rates. The generalized equation is given by[16]

$$
I_{p}=0.446 n F S(D / l) C \beta^{0.5} \tanh \left(0.56 \beta^{0.5}+0.05 \beta\right)
$$

where $\beta\left(=n F v\left(l^{2} / D\right) / R T\right)$ is a dimensionless characteristic time parameter, $l$ means distance of electrode and $F\left(=9.65 \times 10^{4} \mathrm{C} / \mathrm{mol}\right)$ refer to Faraday constant.

\subsection{Sweep rate voltammetry technique}

In a battery system, electrochemical reactions occurs in the electrode through consecutive processes. Such electrochemical reactions can be clearly understood by using a $\mathrm{CV}$ technique-based on the correlations between current, scan rate, and potential. The currents obey the power law, i.e.

$$
i=a v^{b}
$$

where $i$ is current (A), $v$ is scan rate $\left(\mathrm{mV} \mathrm{s}^{-1}\right)$, and $a, b$ are adjustable values. Then $b$ value can be obtained by using simultaneous equations.

The $b$ value can be explained by the sum of the faradaic (diffusion-controlled) and non-faradaic (capacitive) currents. If only diffusion-controlled reaction occurs without any faradaic reaction, the observed current would be proportional to the square root of the scan rate, $v^{1 / 2}$, according to Randles-Sevcik equation, equation (6). On the other hand, the capacitive current (also called "non-faradaic" or "double-layer" current.) follows linearly to the scan rate;

$$
\left|i_{c}\right|=A C_{d} v
$$

where $i_{c}$ is the current for electric double-layer charging, $A$ is surface area of electrode and $C_{d}$ is the capacitance of electric double-layer. As can be seen from the above equation (10), the capacitive current is proportional to scan rate, $v$, therefore, the capacitive current is dominantly measured at a fast scan rate. On the contrary, the recorded current will be

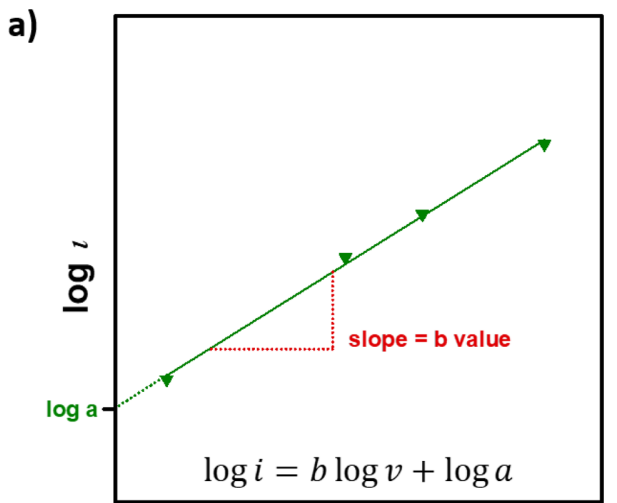

$\log v$ b)

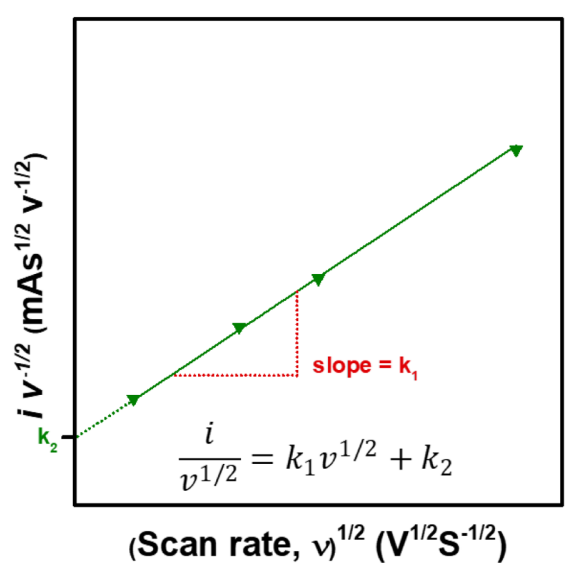

Fig. 4. a) Log-scale plot of peak current dependence on the scan rate, b) The plot from equation (17) for analyzing sweep rate $\mathrm{CV}$ data. 
mainly associated with a diffusion-controlled reaction at a slow scan rate.

Therefore, the $b$ value of total electrochemical reactions composed of diffusion-controlled and capacitive reactions will have values between 0.5 (diffusion-controlled) and 1.0 (capacitive). Set a $\log$ on both side of equation (9), then it can be plotted like Fig. 4a.

$$
\begin{aligned}
& \log i=\log a v^{b} \\
& \log i=\log a+\log v^{b} \\
& \log i=\log a+b \log v \\
& \mathrm{~b}=\frac{\log i-\log a}{\log v}(0.5<b<1)
\end{aligned}
$$

Then, the total current at a specific potential is the sum of both the diffusion-controlled and capacitive reactions.

$$
i=k_{1} v+k_{2} v^{1 / 2}
$$

For analytical purposes, it can be slightly rearranged to,

$$
\begin{aligned}
& \frac{i}{v^{1 / 2}}=\frac{k_{1} v}{v^{1 / 2}}+\frac{k_{2} v^{1 / 2}}{v^{1 / 2}} \\
& \frac{i}{v^{1 / 2}}=k_{1} v^{1 / 2}+k_{2}
\end{aligned}
$$

In equation (15), $k_{1} v$ and $k_{2} v^{1 / 2}$ correspond to the current attributed to capacitive reaction and diffusion-controlled reaction, respectively. Then, $k_{1}$ and $k_{2}$ can be obtained through the linear fit of $\frac{i}{v^{1 / 2}} \operatorname{vs} v^{1 / 2}$ plot from the equation (17) as shown in Fig. $4 b . k_{1}$ represents the slope and $k_{2}$ is the y intercept value. Thus, by determining $k_{1}$ and $k_{2}$, the fraction of the current can be obtained at fixed potential.

\subsection{Estimation of diffusion coefficient through voltammetry}

In order to analyze the kinetics of active materials, we note the Randles-Sevcik equation, equation (6), describing the dependence of the peak current on the scan rate. If the kinetics of electrochemical reactions is only controlled by a single-phase diffusion without phase transition, the oxidation and reduction currents should have a linear dependence on the square root of the scan rate, $v^{1 / 2}$. Like calculating $b$ value, from the relationship of $\mathrm{i}_{\mathrm{p}}$ vs. $v^{1 / 2}$, the apparent diffusion coefficient of $\mathrm{Li}^{+}\left(\mathrm{D}_{\mathrm{Li}}\right)$ can be obtained. If the Randles-Sevcik equation is considered as a linear equation, the plot for current $i_{p}$ as a function of the square root of the scan rate, $v^{1 / 2}$, shows a linear correlation, and all variables can be found as constants. Like sweep rate $\mathrm{CV}$ technique, the diffusion coefficient can be obtained by linear fitting of the slope from the plot $\mathrm{i}_{\mathrm{p}}$ vs. $v^{1 / 2}$;

$$
\begin{aligned}
& \text { slope }=0.446 n F A C\left(\frac{n F D}{R T}\right)^{1 / 2} \\
& D=\left(\frac{\text { slope }}{0.446 n F A C}\right)^{2}\left(\frac{R T}{n F}\right)
\end{aligned}
$$

The above equation is only valid when the phase does not undergo phase transformation during the lithiation/de-lithiation process, i.e., the single-phase diffusion, not multi-phase diffusion. This is an important prerequisite for calculating the diffusion coefficient.

2.6 Determination of specific capacity by voltammetry

When calculating the charge storage in a cyclic voltammogram with an asymmetric shape of the cathodic and anodic peaks, it is usually possible to obtain the integrated area of the cyclic voltammogram. The equation for the specific capacitance estimated from the cyclic voltammogram ( $\mathrm{i}$ vs V plot) is as follows[17],

$$
C(F / g)=\int_{E_{1}}^{E_{2}} i(E) d E / 2\left(E_{2}-E_{1}\right) m v
$$

where $\mathrm{C}$ is the specific capacitance of electrode, in unit $\mathrm{F} \mathrm{g}^{-1} . E_{2}-E_{1}$ is the voltage window and $i(E)$ is the current. $\int_{E_{1}}^{E_{2}} i(E) d E$ is the total charge storage obtained by integration of cathodic and anodic regions in the cyclic voltammograms. $m$ is the mass of active material, and $v$ is the scan rate.

The capacitance is constant in a fixed voltage window and is used to calculate the charge storage,

$$
\Delta Q=C \times \Delta U
$$

where $\Delta Q$ is the capacity ( $\mathrm{C}$ or $\mathrm{mAh}$ ) and $\Delta U$ is the voltage window (V). Further specific capacity (Q) in $\mathrm{mAh} \mathrm{g}^{-1}$ was evaluated from the cyclic voltammograms using the following equation[18], 


$$
Q(\mathrm{mAh} / \mathrm{g})=\int_{E_{1}}^{E_{2}} i(E) d E /(2 \times 3.6) m v
$$

\section{Factors affecting the shape of voltammetric curves}

When evaluating the electrochemical performance, all internal and external factors of the battery should be further considered. Importantly, certain of these factors will directly affect the battery performance because material characteristics, cell configurations and experimental conditions might be various. In this part, we focus on the effects of particle size of active materials, electrolyte concentration, thickness of electrode, scan rate and operating temperature as influential factors for CV measurements.

\subsection{Particle size}

Particle size of active materials is one of the predominant factors directly affecting electrochemical performance. Size reduction induces an increase in the interfacial area of particles (i.e contact area with electrolyte) and shorten the diffusion length of $\mathrm{Li}^{+}$. As a result of enhancement in kinetics of redox reaction, the overall peak current increases at the same scan rate in the cyclic voltammograms. For these reasons, the same active material exhibits different electrochemical performance depending on its particle size. In practice, Yang et al, reported the different

\section{a) Particle size}

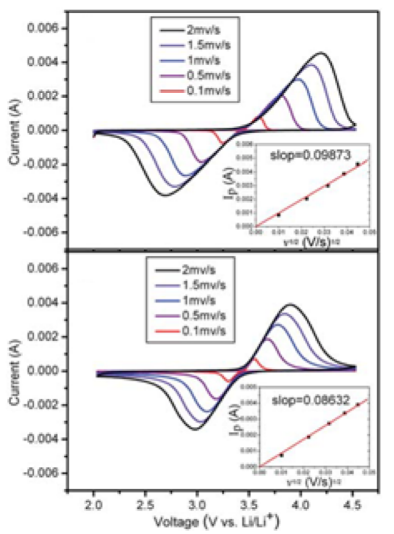

c) Electrode thickness \& scan rate
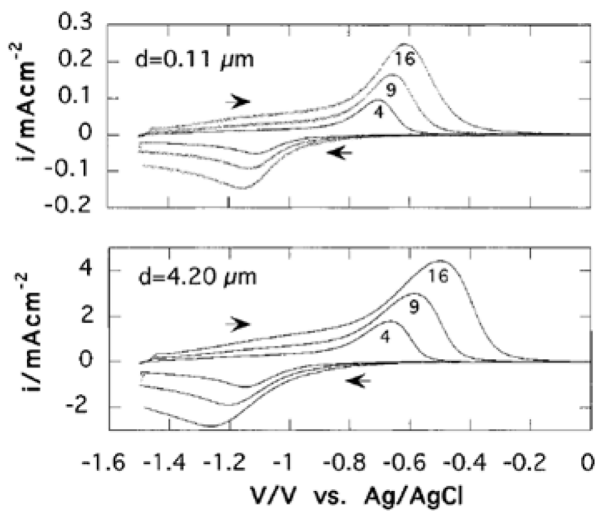

b) Electrolyte concentration

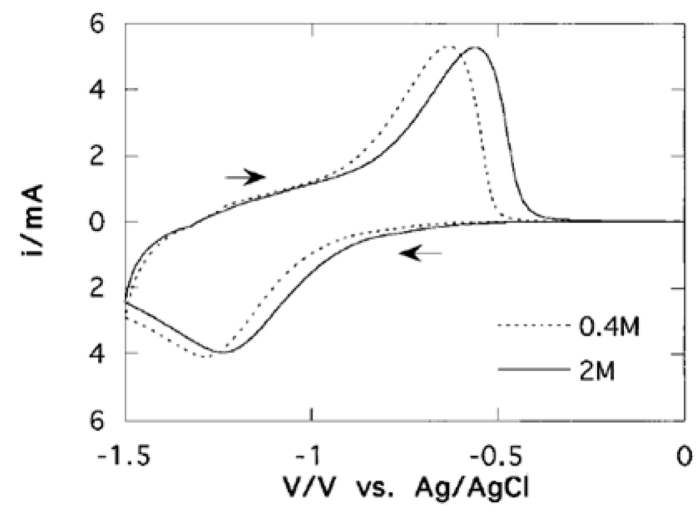

d) Temperature

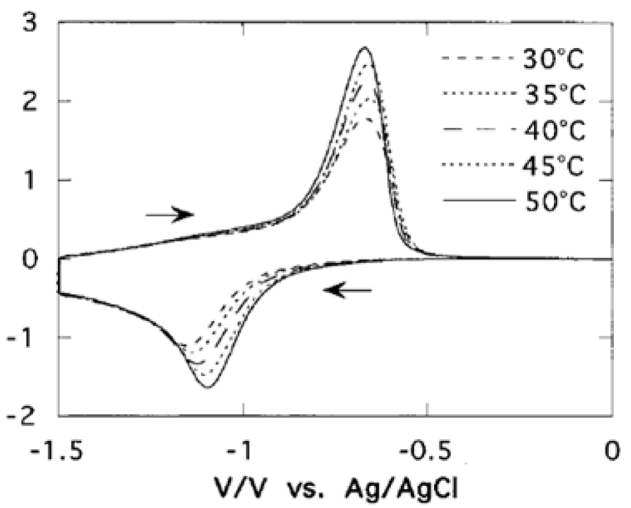

Fig. 5. Factors affecting cyclic voltammetry. a) Particle size. Reprinted with permission from Ref. [19]. Copyright (C) 2010 The Royal Society of Chemistry. b) electrolyte concentration (difference concentration of 2 and $0.4 \mathrm{M}$ ), c) electrode thickness \& scan rate (film thickness of 0.11 and $4.20 \mu \mathrm{m}$ at difference scan rate of 4,9 , and $16 \mathrm{mV} / \mathrm{s}$ ) and d) temperature $\left(30,35,40,45\right.$ and $\left.50^{\circ} \mathrm{C}\right)$. Reprinted with permission from Ref. [20]. Copyright (C) 1997 American Chemical Society. 
electrochemical behaviors in the cyclic voltammograms-obtained from LFP nanoplates and microplates having different particle sizes at various scan rates (Fig. 5a) [19]. Wang et al, also showed the particle size effect of $\mathrm{TiO}_{2}$ on the cyclic voltammograms, in which the contribution of $\mathrm{Li}^{+}$intercalation become smaller but that of capacitance increases by decreasing the particle size [21].

Furthermore, due to the diffusion-controlled $\mathrm{Li}^{+}$ intercalation reaction changed to surface reaction, the overall reaction shows faster kinetic reaction. Therefore, the electrochemical reaction mechanism could be tuned by control the particle size, showing different cyclic voltammograms. Liu et al. also mentioned some electrodes which is composed of various particle sizes of Si exhibit significant differences in cyclic voltammograms [22]. The characteristic alloying reaction peak of $\mathrm{Si}$ with $\mathrm{Li}^{+}$is not clearly detected due to sluggish alloying kinetics of large-particle Si. However, a characteristic alloying reaction peak is more clearly observed in the small-particle $\mathrm{Si}$, which means that the kinetics of the alloying reaction of $\mathrm{Si}$ can be effectively enhanced by the particle size reduction.

\subsection{Electrolyte concentration}

The peak currents are not related to the concentration of $\mathrm{Li}^{+}$in electrolytes fundamentally, and also the peak split between cathodic and anodic peak has nothing to do with the concentration of electrolytes. However, Fig. 5b shows some extent of peak shift with electrolyte concentration. The reaction voltage of the $\mathrm{Li}$ intercalation reaction is related to kinetics of ions in the electrolyte. Basically, electrolytes serve as $\mathrm{H}^{+}, \mathrm{Na}^{+}$, and $\mathrm{Li}^{+}$carriers and do not have a significant effect on the $\mathrm{Li}^{+}$ion diffusion process within the electrode because the charge transfer reactions in the electrode is much slower than those in electrolyte. In the case of aqueous $\mathrm{Na}$ ion battery (SIB), however, the redox peaks corresponding to $\mathrm{Na}^{+}$insertion and extraction are becoming sharper and closer, which indicates faster kinetics at high electrolyte concentrations [23]. As expected by Nernst equation, it is because the equilibrium potential shifts toward more positive direction as the electrolyte concentration increases. Some authors mentioned that the characteristic shifted potential with different electrolyte concentration depends on specific ions, such as $\mathrm{H}^{+}$, $\mathrm{Na}^{+}$, and $\mathrm{Li}^{+}[24,25]$.

\subsection{Scan rate}

In the cyclic voltammograms, peak separation is generally occurs by increasing the scan rate except for reversible process. This peak split is mainly caused by the following reasons; i) potential drops or increase of resistance due to a slow electron transfer or steric hindrance of electrolyte permeation (i.e. porous structure), ii) slow and quasi-reversible or irreversible electrochemical reactions ${ }^{15}$. In principle, the currents induced by the capacitive reaction and the diffusioncontrolled reaction are proportional to $v$ and $v^{1 / 2}$, respectively (equation 6,10 ). This is because the peak current becomes a semi-infinite diffusion process at a high scan rate, and in slow rate condition the peak current is proportional to the square root of the scan rate. Therefore, the general formula including the all of scan rate describe by Aoki et al as mentioned in equation (8). This is the basic principle of the sweep rate CV technique, which can analyze what reaction contributes to charge storage at fixed potential.

Many studies referred to $\mathrm{CV}$ with various scan rates have been reported, as shown in Fig. 5c, and most of the electrode materials tended to follow quasi-reversible processes in which the peak potential increased as the scan rate increased [20,21,2632]. Sweep rate CV technique is used to obtain a clue as to how the mechanism of each electrode proceeds through the electrochemical reaction. This is described in detail in Sections 2.4 and 4.1.

\subsection{Electrode thickness}

As the electrode thickness increases, the peak split between cathodic and anodic peak also increases with scan rate increase, as shown in Fig. 6c. For the thin electrode condition, the entire portion of the electrode is in the uniform state and electrochemically active, so peak split changes are not so large. This means that both the surface and the bulk structure of the electrode have a uniform potential and are homogeneous. However, thick electrodes with scan rate increase show larger peak split compared to thin electrode, since $\mathrm{Li}^{+}$ion diffusion from surface to bulk becomes more difficult [33]. The bulk potential of the electrode depends on distance from surface, which causes potential drop [20].

\subsection{Temperature}

In general, the peak current arising from a diffusion-controlled reaction is significantly affected by 
operating temperature [34]. Since the capacitive current is essentially independent on the temperature, a small peak split at higher temperatures indicates that diffusion-controlled reaction dominantly occurs at the surface of electrode. The rate of electrochemical reaction depends on the $\mathrm{Li}^{+}$diffusion in the lattice of the active materials, and this diffusion process is temperature-sensitive. When potential is applied, the capacitive currents occur and the Helmholtz layer is continually reconfigured on the surface of electrode. The temperature dependence is significantly different between the capacitance of non-faradaic process and the diffusion-controlled reaction of faradaic process. When the peak split decreases at high temperatures, it indicates that the rate of electrochemical reaction becomes faster and the corresponding redox peaks can appear earlier [20] as shown in Fig. 5d.

\section{Applications of voltammetry to the study of $\mathrm{Li}$ battery}

\subsection{Sweep rate voltammetry technique}

A sweep rate CV technique is useful to investigate pseudo-capacitive electrochemical behaviors of cathode materials (e.g. Lithium-free LiF-MO) [30]. Unlike $\mathrm{Li}^{+}$intercalating cathode materials, approximately $94 \%$ of the total capacity is derived from a surface-controlled reaction. As a result, they show excellent power capability maintaining a high discharge capacity of $110 \mathrm{mAh} \mathrm{g}^{-1}$ even at a high current density of $5000 \mathrm{~mA} \mathrm{~g}^{-1}$. The sweep rate $\mathrm{CV}$ technique provides an analysis of the pseudocapacitve electrochemical behavior of the surface-controlled reaction in $\mathrm{LiF}-\mathrm{MnO}$ nanaocomposite.

On the other hand, the reaction mechanism of various anode materials have been extensively studied by using the sweep rate $\mathrm{CV}$ technique. For example, Bruce Dunn group have studied the electrochemical behaviors of $\mathrm{Li}^{+}$intercalating anode materials such as $\mathrm{Nb}_{2} \mathrm{O}_{5}[28,29], \mathrm{TiO}_{2}[20,21,36]$ using the sweep rate $\mathrm{CV}$. Based on equation (17), each contribution of capacitive and intercalation reactions to the total capacity of the anode materials was calculated at a specific voltage, as shown in Fig. $6 c$.

According to the fraction of capacitive current in the whole cyclic voltammograms in Fig. $6 \mathrm{a}$ and 6b, it is evident that the capacitance current and total capacity increase as the particle size decrease. Also, it can be seen that the mechanism changes depending on the change of the sweep rate, that is, the change of the charging time. In the case of $\mathrm{Nb}_{2} \mathrm{O}_{5}$, the $b$ value is estimated to be almost 1.0 at a slow sweep rate $\left(<20 \mathrm{mV} \mathrm{s}^{-1}\right)$, indicating a surface-controlled reaction. On the other hand, the $b$ value decreases to 0.7 at a faster sweep rate $\left(>20 \mathrm{mV} \mathrm{s}^{-1}\right)$. These differences with scan rate can come from ohmic contribution, including active material, SEI resistance, and/or diffusion limitations [37,38]. Since these results depend on the sort and the reaction mechanism of the electrode materials, it is necessary to closely examine the experimental conditions. According to the above paper, the capacitance effect of $\mathrm{Nb}_{2} \mathrm{O}_{5}, \mathrm{TiO}_{2}$, which is known that $\mathrm{Li}$ insertion reaction is the main mechanism, increases greatly depending on various conditions such as electrode thickness, particle size and sweep rate.
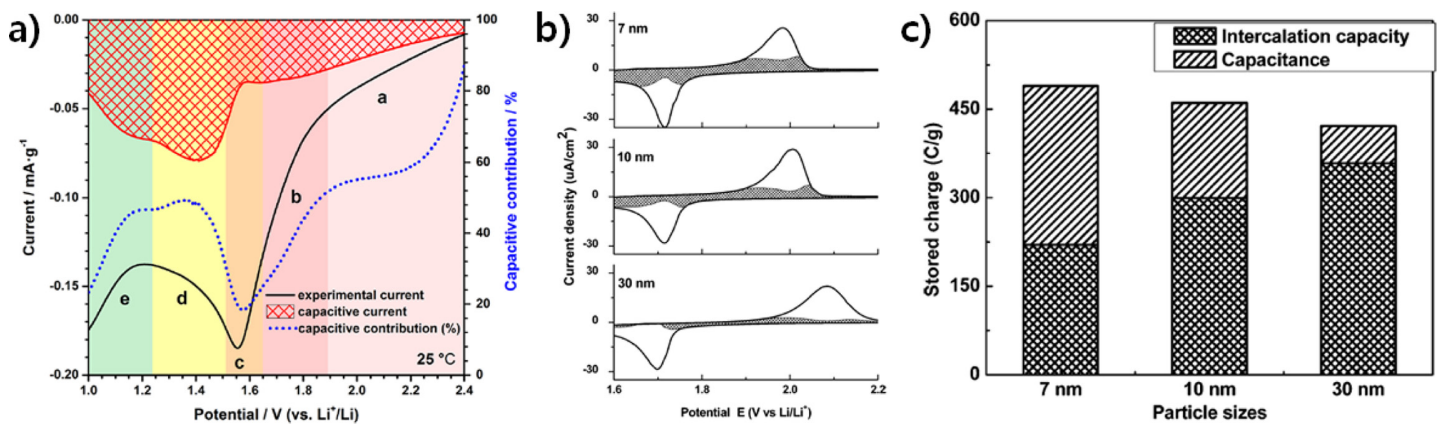

Fig. 6. The example results of comparison of charge storage for $\mathrm{TiO}_{2}$ between intercalation and capacitive contributions. Reprinted with permission from Ref. [35]. Copyright (C) 2017 American Chemical Society. Reprinted with permission from Ref. [21]. Copyright (C) 2007 American Chemical Society. 
Table 1. Comparison of Li ion diffusivity for anode and cathode materials obtained from CV and other analysis (GITT and EIS).

\begin{tabular}{cccc}
\hline \hline Sample & $D_{\mathrm{CV}}\left[\mathrm{cm}^{2} \mathrm{~s}^{-1}\right]$ & $D_{\mathrm{GITT}}$ or $D_{\mathrm{EIS}}\left[\mathrm{cm}^{2} \mathrm{~s}^{-1}\right]$ & References \\
\hline $\mathrm{WO}_{3}$ & $3.79 \times 10^{-11}$ & $3.38 \times 10^{-11}$ & Solid State Ionics 178(2007)259-263 \\
$\mathrm{Si}$ & $5.1 \times 10^{-12}$ & $5.0 \times 10^{-12} \sim 10 \times 10^{-12}$ & Solid State Ionics 180(2009)222-225 \\
$\mathrm{LiCoO}_{2}$ & $2 \times 10^{-13}$ & $\sim 1.0 \times 10^{-12}$ & Journal of Alloys and Compounds 449(2008)300-303 \\
$\mathrm{LiMn}_{2} \mathrm{O}_{4}$ & $10^{-12} \sim 10^{-11}$ & $10^{-12} \sim 10^{-10}$ & Materials Chemistry and Physies 111(2008)149-153 \\
$\mathrm{Li}_{3} \mathrm{~V}_{2}\left(\mathrm{PO}_{4}\right)_{3}$ & $10^{-10} \sim 10^{-11}$ & $10^{-8} \sim 10^{-13}$ & Electrochimica Acta 55(2010)2384-2390 \\
\hline
\end{tabular}

\subsection{Estimation of diffusion coefficients}

The sweep rate CV technique can be used for calculating the diffusion coefficients of both cathode and anode materials. In practice, it has been extensively used for various cathode materials such as layered [39], spinel [40], olivine [19,41], and even LiFMO [30] nanocomposite. In addition, the overall average $\mathrm{Li}^{+}$diffusivity of various anode materials also can be estimated. To clarify the reaction mechanism, it needs to analyze $\mathrm{Li}^{+}$diffusivity when each phase transition occurs in the active materials. For example, spinel $\mathrm{LiMn}_{2} \mathrm{O}_{4}$ exhibits multiple phase transitions, $\mathrm{LiMn}_{2} \mathrm{O}_{4} \rightarrow \mathrm{Li}_{0.5} \mathrm{Mn}_{2} \mathrm{O}_{4}$ and $\mathrm{Li}_{0.5} \mathrm{Mn}_{2} \mathrm{O}_{4}$ $\rightarrow \lambda-\mathrm{MnO}_{2}$, during the charge process. The corresponding $\mathrm{Li}^{+}$diffusivities are calculated to be $\mathrm{MnO}_{2}$ are $4.63 \times 10^{-12}$ and $1.04 \times 10^{-11} \mathrm{~cm}^{2} \mathrm{~s}^{-1}$, respectively, and in the discharge process are $2.88 \times 10^{-12}$ and 1.68 $\times 10^{-12} \mathrm{~cm}^{2} \mathrm{~s}^{-1}$, respectively. The $\mathrm{Li}^{+}$diffusivity values obtained by the sweep rate $\mathrm{CV}$ are comparable to the diffusion coefficient calculated from PITT $(1.9 \times$ $10^{-12}$ to $\left.8 \times 10^{-11} \mathrm{~cm}^{2} \mathrm{~s}^{-1}\right)$. Note that the sweep rate $\mathrm{CV}$ can be used for estimation of the diffusion coefficients in the single-phase reaction. However, the values of the diffusion coefficients obtained from the materials which undergo phase transition need to be carefully approached for their reliability.

On the other hand, some authors reported the difference of diffusion coefficient of active materials according to the analytical methods such as CV and EIS or GITT. Ding et al. [42]. claimed that different diffusion coefficients were obtained from nano-Si with values of $5.1 \times 10^{-12} \mathrm{~cm}^{2} \mathrm{~s}^{-1}$ and $10^{-12} \mathrm{~cm}^{2} \mathrm{~s}^{-1}$ by $\mathrm{CV}$ and EIS measurements, respectively. The diffusion coefficient measured by EIS is about 5 times lower than one calculated from GITT. A significant difference of diffusion coefficients of $\mathrm{WO}_{3}$ were also obtained by CV and GITT. The difference is even more pronounced at high scan rates (above $2 \mathrm{mV} \mathrm{s}^{-1}$ ), because the Randles-Sevcik method tends to generate large potential gradients in the active material, which greatly overestimates the diffusion coefficient. In addition, the characteristic is attributed to different equilibrium conditions between analytical methods. In practice, the EIS measurement is conducted under more equilibrium conditions (more relaxation time) compared with GITT. However, both methods show similar variation of $\mathrm{Li}^{+}$diffusivity in silicon. The diffusion coefficient for single-phase reaction is more reliable when the slope of $\log$ (peak current) vs. $\log$ (scan rate) is 0.5 (i.e. diffusion-controlled). When $\mathrm{Li}^{+}$ transport is governed by the cell resistance, such relationship will be invalidated which is called as "cellimpedance-controlled" situation [43].

\subsection{Stepwise voltage window technique}

An useful application of the stepwise voltage window technique is an anionic redox analysis of LR$\mathrm{NMC}\left(\mathrm{Li}\right.$-rich $\mathrm{Li}_{1.2} \mathrm{Ni}_{0.13} \mathrm{Mn}_{0.54} \mathrm{Co}_{0.13} \mathrm{O}_{2}$ ) [44]. To clarify the reaction mechanism of Li-rich cathode materials, a lot of research has been carried out on anionic redox as well as cationic redox reactions in these days. The measurement proceeds with an increase of potential cut-off starting from a small value, gradually increasing to the existing voltage window after a few formation cycles. For example, in a voltage window from 2.0 to $4.8 \mathrm{~V}$, the potential cut-off gradually increases from 2.0 to 3.5 , to 3.7 , to 3.9 , and finally reaches 2.0 to $4.8 \mathrm{~V}$. As shown in Fig. 7a, the voltage hysteresis appears noticeably beyond $4.1 \mathrm{~V}$ (red curves) when the charge voltage window starts to increase gradually from $2.0 \mathrm{~V}$. In the corresponding $d Q / d V$ curve, it was observed that the charge capacity beyond $4.1 \mathrm{~V}$ is only partially recovered on reduction at a high voltage and the remaining reduction took place at a low voltage (below 3.6 V). The charge compensation above $4.1 \mathrm{~V}$ (red curves) can be assumed to be the contribution of anionic oxidation, since the cationic oxidation is nearly finished at about 
a

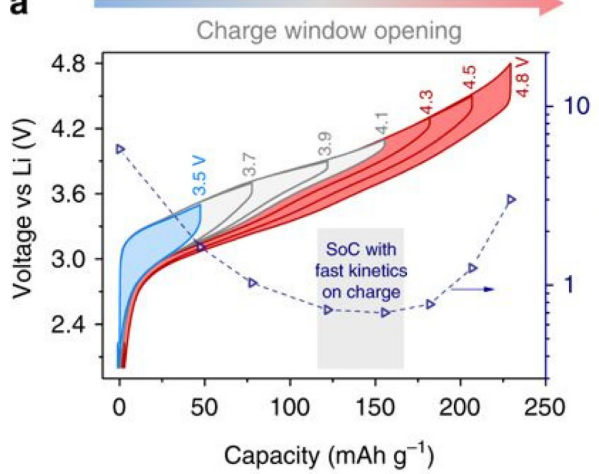

b

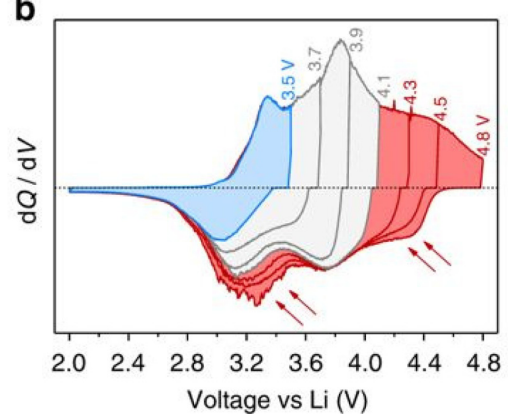

C
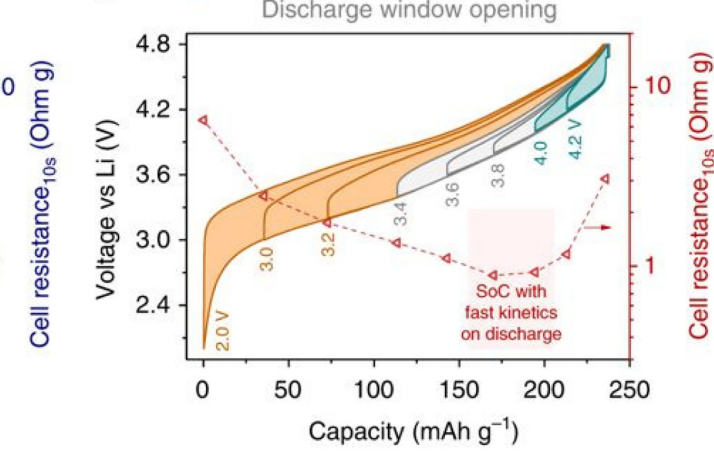

d

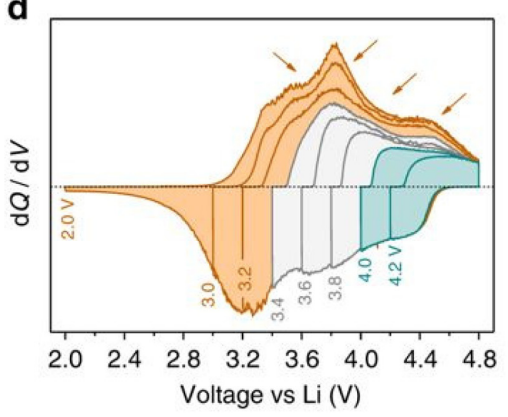

Fig. 7. Electrochemical analysis of LR-NMC cathode materials. a) and c) charge and discharge voltage profiles, respectively, b) and d) corresponding stepwise $d Q / d V$ curves. Reprinted with permission from Ref. [44]. Copyright (C) 2017 Springer Nature.

4.1 V. Similarly, in Fig. 7c and 7d, anionic redox reaction is clearly visible at reductions of less than $3.4 \mathrm{~V}$. It shows that the voltage hysteresis due to anionic redox reaction appears asymmetrically during the charge-discharge process.

\section{Conclusions}

Cyclic voltammetry (CV) is an electrochemical analysis technique that is widely used in various fields of electrochemistry, enabling the analysis of electrochemical redox reactions of active materials by recording the current according to voltage change. It is useful for determining the redox reaction potential, and furthermore, understanding the thermodynamics and kinetics of the electrochemical systems in detail. It was also found that different cyclic voltammograms were derived from various factors such as particle size of active materials, thickness of electrode, electrolyte concentration, operating temperature and scan rate of experimental conditions. In addition, it can be used for quantitative analysis of the current attributed to surface- and diffusion- controlled reactions, and the $\mathrm{Li}^{+}$diffusivity of each reaction by varying the scan rate. Furthermore, anionic redox, which is mainly studied in Li-rich cathode materials, can also be analyzed through a stepwise voltage window technique.

In this mini-review, we briefly introduce the fundamental principle of cyclic voltammetry, and its applications for research in LIB. As technology is increasingly sophisticated, the role of state-of-the-art analytical technology is becoming more important. However, it should not be blindly pursued without an understanding of basic electrochemistry. For a better understanding of electrochemistry and batteries, cyclic voltammetry will provide us plenty of ideas about the overall electrochemical mechanism.

\section{Acknowledgment}

This work was supported by the National Research Foundation of Korea (NRF) grant funded by the Korea government (MSIP) (No. NRF-2017R1A4A1015770). 
This work was also supported by the National Research Foundation of Korea (NRF) grant funded by the Korea government (MSIP) (No. NRF2019R1A2C2003731). W.C. acknowledges NRF Grant funded by Korean Government (NRF2016H1A2A1910157-Global Ph.D. Fellowship Program).

\section{Supporting Information}

Supporting Information is available at https:// doi.org/10.33961/jecst.2019.00619

\section{References}

[1] W. Lee, S. Muhammad, C. Sergey, H. Lee, J. Yoon, Y.M. Kang, W.-S. Yoon, Angew. Chemie Int. Ed., 2019.

[2] J.H. Um, K. Palanisamy, M. Jeong, H. Kim, W.-S. Yoon, ACS Nano, 2019, 13, 5674-5685.

[3] S. Muhammad, S. Yun, K. Palanisamy, H. Kim, W.S. Yoon, J. Power Sources, 2019, 423, 323-330.

[4] H. Kim, D.S. Yang, J.H. Um, M. Balasubramanian, J. Yoo, H. Kim, S. Bin Park, J.M. Kim, W.S. Yoon, J. Power Sources, 2019, 413(December 2018), 241-249.

[5] Y. Kim, D.S. Kim, J.H. Um, J. Yoon, J.M. Kim, H. Kim, W.S. Yoon, ACS Appl. Mater. Interfaces, 2018, 10(35), 29992-29999.

[6] W. Lee, S. Muhammad, T. Kim, H. Kim, E. Lee, M. Jeong, S. Son, J.H. Ryou, W.S. Yoon, Adv. Energy Mater, 2018, 8, 1701788.

[7] H. Lindström, S. Södergren, A. Solbrand, H. Rensmo, J. Hjelm, A. Hagfeldt, S.-E. Lindquist, J. Phys. Chem. B, 1997, 101(39), 7710-7716.

[8] D. J. Chesney, J. Am. Chem. Soc., 1996, 118(44), 10946-10946.

[9] K. Brainina, E. Neyman, 1994, Electroanalytical Stripping Methods, Wiley.

[10] D.A. Skoog, F.J. Holler, S.R. Crouch, 2017, Principles of Instrumental Analysis, Cengage learning.

[11] E. Talaie, P. Bonnick, X. Sun, Q. Pang, X. Liang, L.F. Nazar, Chem. Mater, 2017, 2990-105.

[12] W.F. Potyrailo, R. A.; Maier, CRC Press, 2006, Combinatorial and High- Throughput Discovery and Optimization of Catalysts and Materials.

[13] D.A.C. Brownson, D.K. Kampouris, C.E. Banks, Chem. Soc. Rev, 2012, 41(21), Graphene Electrochemistry: Fundamental Concepts through to Prominent Applications.

[14] H. Matsuda, Y. Ayabe, Zeitschrift für Elektrochemie, Berichte der Bunsengesellschaft für Phys. Chemie, 1955, 59(6), 494-503.

[15] M. Takahashi, S. ichi Tobishima, K. Takei, Y. Sakurai, Solid State Ionics, 2002, Solid State Ionics.

[16] K. Aoki, K. Tokuda, H. Matsuda, J. Electroanal. Chem.,
1983, 146(2), 417-424.

[17] W. Chen, Z. Fan, L. Gu, X. Bao, C. Wang, Chem. Commun., 2010, 46(22), 3905-3907.

[18] A. Krause, P. Kossyrev, M. Oljaca, S. Passerini, M. Winter, A. Balducci, J. Power Sources, 2011, 196(20), 8836-8842.

[19] S. Yang, X. Zhou, J. Zhang, Z. Liu, J. Mater. Chem., 2010, 20(37), 8086-8091.

[20] H. Lindström, S. Södergren, A. Solbrand, H. Rensmo, J. Hjelm, A. Hagfeldt, S.-E. Lindquist, J. Phys. Chem. B, 1997, 101(39), 7717-7722.

[21] J. Wang, J. Polleux, J. Lim, B. Dunn, J. Phys. Chem. C, 2007, 111(40), 14925-14931.

[22] W.-R. Liu, Z.-Z. Guo, W.-S. Young, D.-T. Shieh, H.-C. Wu, M.-H. Yang, N.-L. Wu, J. Power Sources, 2005, 140(1), 139-144.

[23] W. Wu, S. Shabhag, J. Chang, A. Rutt, J.F. Whitacre, J. Electrochem. Soc., 2015, 162(6), A803-A808.

[24] G. Redmond, D. Fitzmaurice, 1993, 1426-1430.

[25] G. Rothenberger, D. Fitzmaurice, M. Gratzel, 1992, 5983-5986.

[26] T.-C. Liu, J. Electrochem. Soc., 1998, 145(6), 1882.

[27] Z. Chen, V. Augustyn, X. Jia, Q. Xiao, B. Dunn, Y. Lu, ACS Nano, 2012, 6(5), 4319-4327.

[28] X. Wang, G. Li, Z. Chen, V. Augustyn, X. Ma, G. Wang, B. Dunn, Y. Lu, Adv. Energy Mater, 2011, 1(6), 10891093.

[29] H.D. Abruña, M.A. Lowe, P.-L. Taberna, B. Dunn, V. Augustyn, S.H. Tolbert, J.W. Kim, J. Come, P. Simon, Nat. Mater., 2013, 12(6), 518-522.

[30] S.K. Jung, H. Kim, M.G. Cho, S.P. Cho, B. Lee, H. Kim, Y.U. Park, J. Hong, K.Y. Park, G. Yoon, W.M. Seong, Y. Cho, M.H. Oh, H. Kim, H. Gwon, I. Hwang, T. Hyeon, W.S. Yoon, K. Kang, Nat. Energy, 2017, 2(2), 4-12.

[31] Y.S. Lee, K.S. Ryu, Sci. Rep., 2017, 7(1), 1-13.

[32] H. Kim, J. Hong, Y.-U. Park, J. Kim, I. Hwang, K. Kang, Adv. Funct. Mater., 2015, 25(4), 534-541.

[33] K.-C. Tsay, L. Zhang, J. Zhang, Electrochim. Acta, 2012, 60428-436.

[34] O.P. Siclovan, G. Zappi, G.L. Soloveichik, ECS Electrochem. Lett., 2014, 3(12), H41-H43.

[35] X. Hua, Z. Liu, M.G. Fischer, O. Borkiewicz, P.J. Chupas, K.W. Chapman, U. Steiner, P.G. Bruce, C.P. Grey, J. Am. Chem. Soc., 2017, 139(38), 13330-13341.

[36] M. Opitz, J. Yue, J. Wallauer, B. Smarsly, B. Roling, Electrochim. Acta, 2015, 168125-132.

[37] M. Park, X. Zhang, M. Chung, G.B. Less, A.M. Sastry, J. Power Sources, 2010, 195(24), 7904-7929.

[38] S. Ardizzone, G. Fregonara, S. Trasatti, Electrochim. Acta, 1990, 35(1), 263-267.

[39] S.B. Tang, J. Alloys Compd., 2008, 449(1-2), 300-303.

[40] S.B. Tang, M.O. Lai, L. Lu, Mater. Chem. Phys., 2008, 111, 149-153.

[41] X.H. Rui, N. Ding, J. Liu, C. Li, C.H. Chen, Electrochim. Acta, 2010, (55), 2384-2390. 
[42] N. Ding, J. Xu, Y.X. Yao, G. Wegner, X. Fang, C.H. Chen, I. Lieberwirth, Solid State Ionics, 2009, 180, 222225.

[43] H.C. Shin, S. Il Pyun, Electrochim. Acta, 2001, 46(16),
2477-2485.

[44] G. Assat, D. Foix, C. Delacourt, A. Iadecola, R. Dedryvère, J.M. Tarascon, Nat. Commun., 2017, 8(1). 